\title{
Designing a conceptual framework to enhance the success of Business Process Reengineering in hospital environments based on holonic structures
}

\author{
Alireza Movassagh \\ Industrial engineer \\ Iran University of science and technology \\ Narmak, Tehran, Iran, 1684613114 \\ Email: alireza.movassagh@gmail.com
}

\begin{abstract}
In order to tackle the numerous problems facing with service management in healthcare industry, various quality improvement (QI) techniques are used for improving the quality of services in this section. Among with these techniques, Business Process Re-engineering (BPR) has more potential to solve these challenges, due to its focus on fundamental changes. However this approach, in turn, have a significant executive challenges and problems, especially in change process, which is rooted in sever hierarchical structure in these environments particularly in hospitals. Hence, whereas the process-oriented structure is one of the critical success factors in BPR implementation, this structure cannot be completely employed in hospital environments. To overcome mentioned obstacle, this research aims to develop a new organizational structure in the process of BPR implementation in hospitals, based on "Holonic concepts" as a kind of nonhierarchical structures. In his model, each care services is met by means of some "care holons" that each of them can be adapted according to structural requirements of related services and consequently can bring advantages of both vertical and horizontal organizational structures.
\end{abstract}

Keywords: BPR, Hospital process, Holonic structures, Healthcare management.

\section{Introduction:}

Healthcare industry, nowadays is suffering from too many obstacles and challenges. Long waiting times, lack of adequate access to health services and poor quality of services are the examples of mentioned problems. To overcome these difficulties, new managerial approaches such as continuous improvement, Lean techniques, TQM, Business Processes Reengineering (BPR) and similar ones in both theoretical and practical studies have been developed to identify and resolve bottlenecks and redundant or prone to error activities for enhancing the quality of healthcare services ${ }^{1}$.

Naturally, Quality Improvement (QI) techniques such as Total Quality Management (TQM ), Continuous Quality Improvement
(CQI), will lead to minor improvements. Instead, because the focus of the BPR is on the sweeping changes in the business processes, it has more potential to achieve efficient and stable improvements which are achieved through eliminating and redesigning of non-value added processes and inefficient organizational structure $^{2}$. Based on these characteristics, it seems that this approach especially in environments that require radical changes, is quite a good option and therefore, can be regarded as a suitable approach for coping with long-term problems in healthcare industry ${ }^{3}$.

But, based on literature review which will presented in follow, fully implementation of this approach, isn't simple and too many factors should be considered to guarantee the efficieny of this method ${ }^{3}$. 
As it turns out, the hardest part is to manage the change process in an effective way, because these environments like hospitals, traditionally have a hierarchical structure that should be converted to interdisciplinary team works, that this change is mostly a time-consuming process ${ }^{4}$. According to literature, this factor is one of the main reasons of BPR failures in hospitals that despite the considerable researches, it is still challenging problems in this topic ${ }^{5}$.

Consequently, main objective of this study is developing the new organizational structure for solving mentioned problem. Due to the flexibility and adaptability of a "Holonic structure", it can be considered as a good choice for BPR implementation in hospital environments and eliminating or at least, mitigating the related structural challenges by offering the benefits of both hierarchical and non-hierarchical approaches.

The rest of the paper is organized as follows: Next section, presents the BPR Literature review in hospitals. In third section, holonic concepts and their capabilities and potentials in turbulent environments, is introduced. In fourth section, proposed structure is discussed and final section, concludes the paper along with some suggestions for future works.

\section{BPR in hospital environments}

A look at the BPR History shows that this approach has been used since the early 1990's which was resulted in significant improvements in productivity through ameliorating in time, cost and quality of services and accordingly enhance the customer satisfaction ${ }^{6}$. Too many organizations all over the word have implemented BPR in different industries such as information and communication technology (ICT), healthcare, manufacturing, production, construction, education and public services ${ }^{7,8}$. According to what has been presented, addressing the BPR approach in healthcare, has the compelling reasons and therefore, a lot of experiences in this field can be seen in the literature.
However, from some standpoints, BPR couldn't meet expectations due to some inherent weak points. One of the obstacles facing the BPR implementation process, is that health environments commonly have vertical and topdown structure, whereas one of the requirements of the successful BPR implementation is team working, which requires process-based and flat structures. Thus, the conflict between traditional structures in hospitals and favorable structure for the proper implementation of this approach can be identified.

Thus, considering these shortages, BPR may lead to deprive the motivation of employees, because they cannot be involved in planning and change management process.

According to related researches, healthcare environments have an extremely hierarchical structures, which should be converted to interdisciplinary team works, which is mainly a long-lasting process. According to this studies, hierarchical structures are more preferable in healthcare, which in turn it makes communication difficult, because all the requirements of a hierarchical structure should be considered ${ }^{7}$.

For solving this problem, some studies have used combined methods, like applying modified BPR by means of "Lean concepts"9. McNulty and Ferlie (2004) reported a real example of the failure of a BPR project in a hospital. They argued that main reason of this failure, was that the processes were implemented in a functional structure $^{10}$. Other researchers believe that it is impossible to create fully process oriented structure in hospitals. For example, Wihlman et al. (2010), has suggested that a combination of both horizontal and vertical structure, would be the desirable arrangement ${ }^{5}$.

In similar survey, Palmberg (2010) also observed in her case studies that the best way is to combine functional and process-oriented organization structure in a matrix form. Although by taking this approach, main problem which is tied to complete hierarchical structures, partly can be reduced, Of course, this leads to new problem and may result in increasing 
conflicts between functional and process managers ${ }^{12}$.

From what has been explained above, literature study shows that despite the importance of this topic, appropriate organizational structure is still one of the main areas of research in hospital BPR domain ${ }^{13}$.

\section{Gap analysis}

According to literature review, a research gap can be recognized in hospital BPR researches. On the one hand, studies indicate that growing importance of health, has led to emphasis on quality improvement concepts especially on BPR. But it seems that this approach didn't have considerable success, due to contradiction between desired and conventional organizational structure in BPR implementation process.

Utilization of "matrix organization" is one of the latest solutions which was suggested by Palmberg (2010) ${ }^{12}$. In this research, the role and importance of flexible structures has been clearly emphasized, but no clear explanation and implementation mechanism, has been provided.

Accordingly, gap analysis resulting from literature review is summarized schematically in figure1.

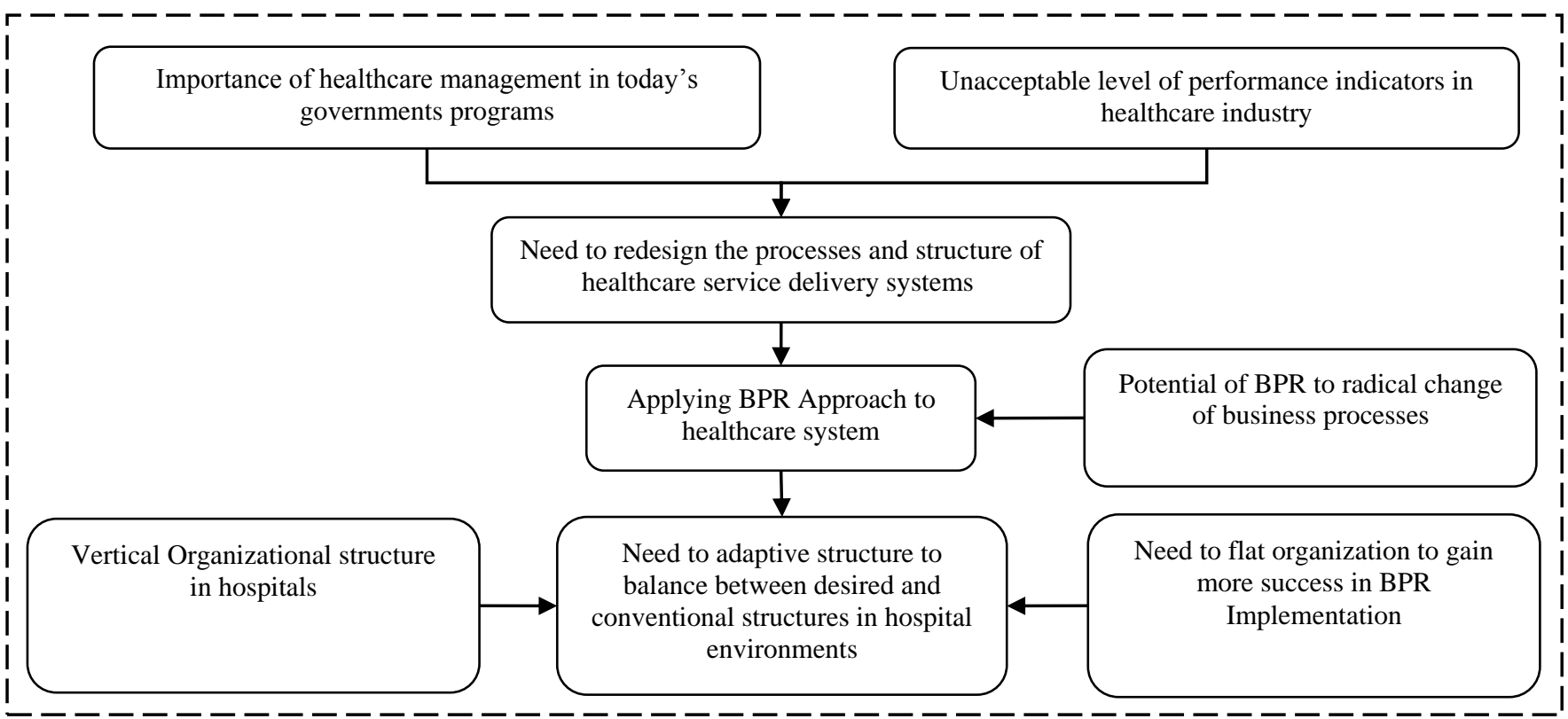

Fig. 1. Gap Analysis (Developed for this study)

In next section, "holonic systems" as an aspects of flexible structures, is introduced and their potential to solve this problem is discussed.

\section{Holon, Holarchy and Holonic Structures}

As mentioned in the introduction, this paper tries to solve the mentioned problem by using holonic structures. Hence, firstly, the "Holon" as a main element of a holonic systems is introduced and in continue, the main characteristics of a holonic structures in contrast with classical ones are discussed ${ }^{14}$.

The word "holonic" derived from term "holon" which is a mixture of "hol" that implies on whole, and the suffix "on" which implies on subdivision. Therefore, Holon $=$ Whole + Part. In holonic viewpoint, each object can be considered as a holon that consists of multiple smaller subsets, and at the same time is a subset of a larger set ${ }^{14}$. 
Although this approach had been proposed in social issues, but it found wide applications in the analysis of complex systems in other contexts. For example, a supply chain, is a part of a larger supply chain and simultaneously, consists of several particles or elements, such as some retailers, wholesalers or factories.

One of the holon's important characteristics is that a holon is "recursive" and given to its utility to this flexibility, this approach, has been widely used to solve complicated industrial and managerial problems such as production scheduling, supply chain management, traffic control and energy distribution systems ${ }^{15-22}$.

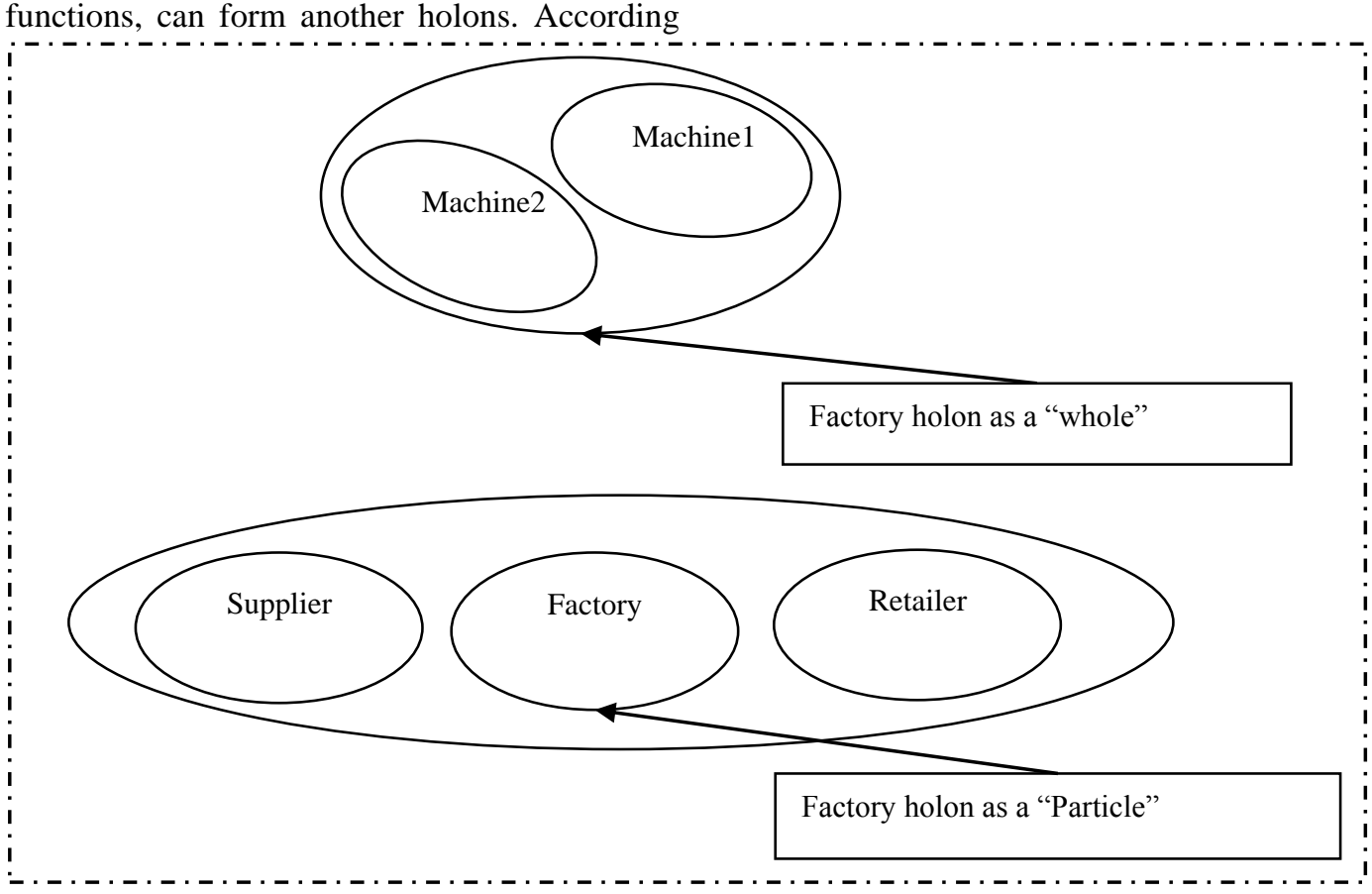

Fig.2. An example of a "holon" (Developed for this study)

Not only in hard problems, but also in soft subjects such as human resource management, this approach has been investigated ${ }^{23}$. For example, Hongyi et al (2001), have shown the potential of this structures in human resource management. Their study, showed the significant improvement in human performance with new system in contrast with classical (hierarchical) structures ${ }^{23}$.

Beside the concept of the holon, new impression related to structure of holons has been emerged which named "Holarchy". In this subject, Mella. P(2009), says that" holons must necessarily be included in other holons in a typical vertical arrangement, with progressive accumulation and forming a nested hierarchical order called a holarchy, which can be represented as an arborized structure (turned upside down to fit this particular context) whose branches become larger at each successive hierarchical level "16. figure3, presents a holarchy and hierarchy structures. 


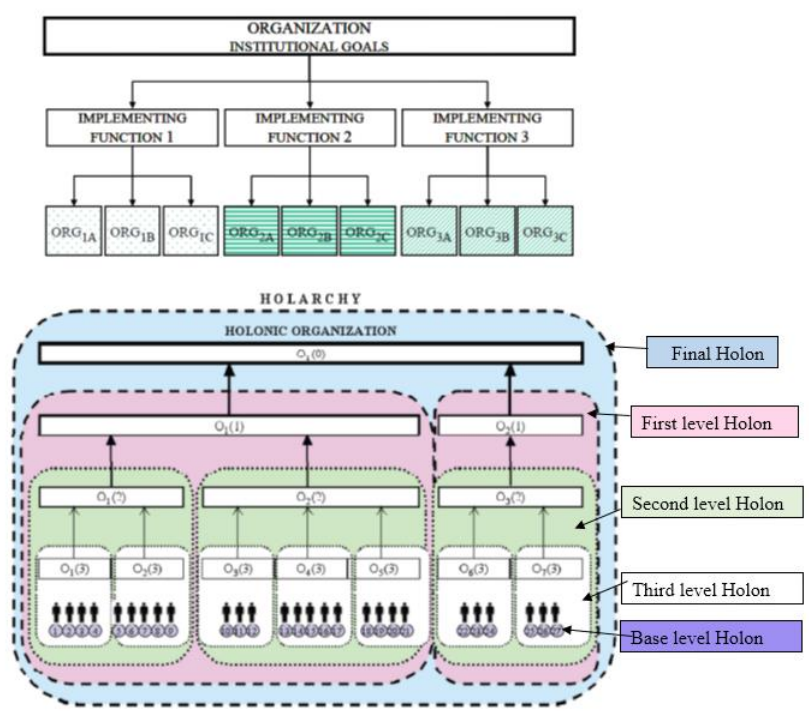

Fig.3. Holarchy vs. or Hierarchy Organization ${ }^{16}$

Adaptability and flexibility of these structures, is a main motivation to use them in mentioned problem in hospital environments. In fact, experiences have shown that effective implementation of BPR, requires the restructuring from vertical organization to flat one. But, from other point of view, this transforming, may lead to employee's resistance, especially in hospital environments that conventionally have too rigid vertical hierarchies. Also according to nature of health services, it is impossible to implement fully flat structures. Therefore, an adaptive structure is needed to maintain a balance between BPR requirements and hospital characteristics.

\section{Proposed structure}

According to the gap analysis, in this section, a new structure based on holonic concepts will be developed in two following steps:

\subsection{Mapping care services with process requirements}

As can be concluded from literature, hierarchical structure can function well in environments without or with less fluctuations. On the other hand, decentralized structures are fully flexible and according to this characteristic, they can be used in more turbulent conditions.
Therefore, one of the main points to develop an appropriate structure, is distinguishing the requirements of each process that directly depends on the type of health services. In another word, to develop an operational model for improving BPR effectiveness in hospitals, it is necessary to distinguish the clear map between the services which are delivered in these environments and their related processes requirements.

Accordingly, if the effective variables on processes are manageable, hierarchical structures is appropriate. But otherwise, the structure should be amenable to decentralized one, to bring good flexibility in confronting with environmental fluctuations and unstable situations (fig4).

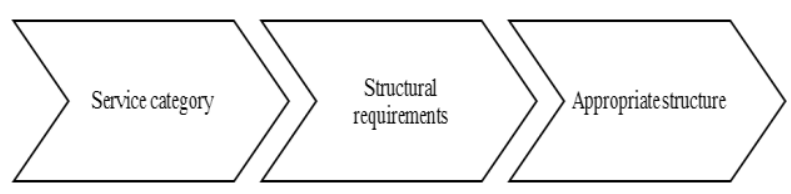

Fig. 4. Decide about organizational structure (Developed for this study)

To clarify it more, an example is presented in table (1). In this example, care services of hospitals, have been divided to 3 categories and appropriate structure is presented for each of them, based on their inherent requirements. 
Table 1: Identifying the suitable structural characteristics of care services (Developed for this study)

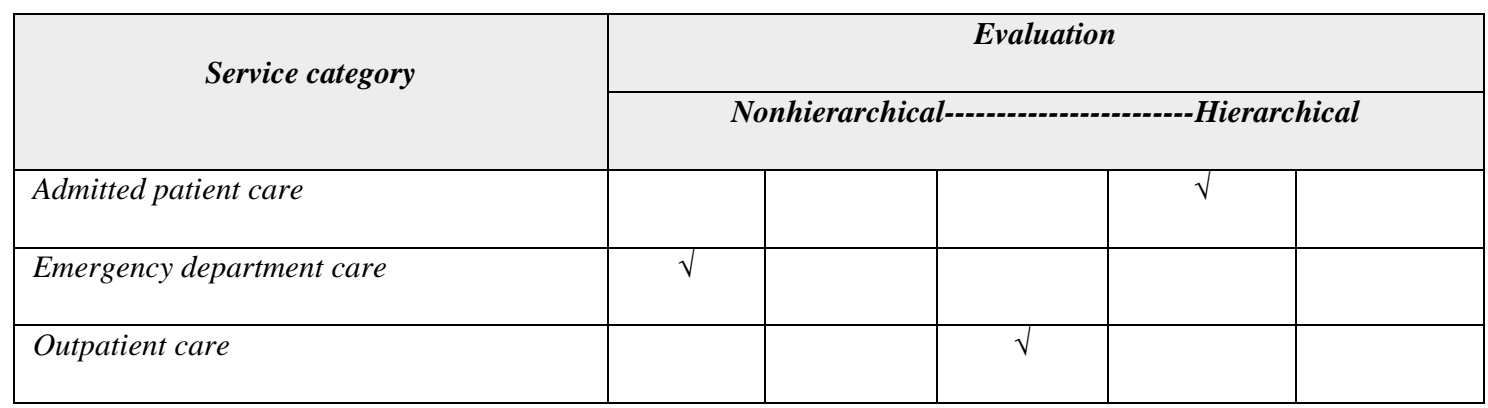

In this table, for each service, appropriate structure is suggested, according to their necessities. For example, in the case of emergencies, flexibility and fast response is very important, whereas it's not more important for admitted patient care. Therefore their structures are not the same.

To sum up, in order to design an appropriate structure, it is critical to develop a mechanism to determine the process requirements. This mechanism, maps the care services and related process requirements and structures.

\subsection{Creating "care holons"}

After determining required structure for each service which is explained in last section, desired holonic structure should be shaped. In this structure, according to each situation and required service, a "care holon" will be created that each of them is consists of humans with needed expertise to complete one task. Whenever condition changes, this holon is demolished and according to new service characteristics and needed structure, different holons will exist. In each condition, structure of holons can be inclined to hierarchical or nonhierarchical, which is determined by requirements of related services.

Hence, this structure is neither rigid hierarchical structure, nor quite decentralized structure. But instead, in this adaptive structure, some "care holons" with hierarchical or nonhierarchical (depends on demands of services) are shaped for doing specific task, and then reshape to complete another task. Figure 5, depicts a sample to clear this mechanism. 


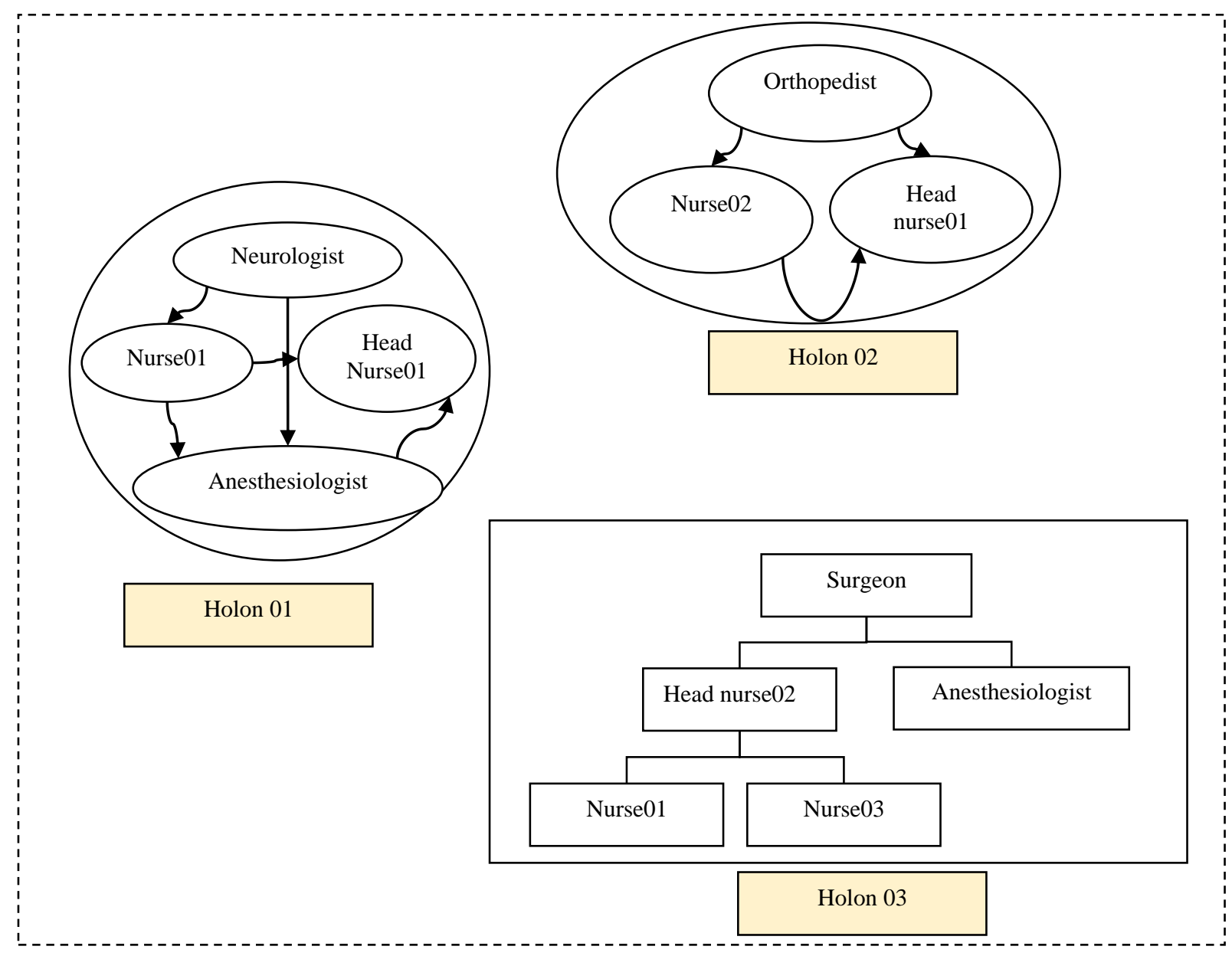

Fig. 5. Creating care holons (Developed for this study)

For example, suppose that for a new arrived care service, suitable structure is heterarchical. Therefore, a "Holon1" is shaped by means of need expertises in heterarchical form. After this situation, this holon will be destroyed and each holon members, become a member of other holons ("Holon2 and 3") to complete another task.

\section{Conclusion and future works}

Although it is obvious that implementing BPR or more generally, creating a process based healthcare, can decrease the problems of healthcare environments, this approach has not resulted in considerable outputs in hospital environments. Reviewing the literature shows that one of the obstacles which affects the effectiveness of this approach, is due to the vertical organizational structure of these environments.

Regarding the flexibility and adaptability of nonhierarchical structures, it seems that these structures can be a good choice for mitigating the mentioned challenge. These structures, based on the requirements of the different environments, can offer the benefits of both hierarchical and non-hierarchical approaches.

Therefore, in this article, a mechanism, based on this concepts has proposed. In this approach, each health service, is done by means of specific 
execution structures named "care holons". These holons, according to requirements of each demanded service, shape themselves in the arrangement of hierarchical or heterarchical and accordingly complete their tasks, based on the relations which is determine in the structure of holon. After finishing one task, this holon is reshaped to complete another task, by means of new members. With this structure, disadvantages and challenges of hierarchical and rigid structures, can be reduced and likelihood of success in BPR implementation will be increased. Hence, the main finding of this research is showing the capabilities of nonhierarchical structures (especially holonic ones) to increase the success of BPR in hospital environments, which can be considered as a new concepts in BPR academic and practical researches. Moreover, this finding not only in hospitals or other sectors of healthcare industry, but also in other environments and industries in which, selection of organizational structure is critical to reach the desired results of BPR, can be considered .

Because the implementation of this system is time consuming and requires training to individuals, it was impossible to put this model in real word, which was the main limitation of this research. It is noticeable that to gain more advantage from implementation of this model, this approach should be encouraged by top level management to develop the required team working culture.

According to outcomes and limitations of this research, there are some subjects which can be followed in future researches. Firstly, this mechanism should be implemented in real cases that possible problems become clear. Because in proposed model, flexibility in relations is needed, implementation can illuminate the requirements of employees in both hierarchy and heterarchy structures such as educational requirements. Also, because in this model, each person can act in different positions, it requires the modification in job descriptions. Consequently, performance measurement models and indicators should be adapted based on these new structures. Finally, categorizing the care services to identify suitable structures, can be done with more details, in more categories and in more levels.

\section{References:}

1. Khodambashi.S,'Business Process ReEngineering Application in Healthcare in a relation to Health Information Systems", Procardia Technology 9, 2013, 949 - 957.

2. Patwardhan, A. and D. Patwardhan, "Business process re-engineering-savior or just another fad? One UK health care perspective". International Journal of Health Care Quality Assurance, 21(3), 2008, 289-296.

3. Patwardhan.A, Patwardhan.D,"Business Process re-engineering saviour or just another fad?", International Journal of Health Care Quality asseurance, 21,3,2008.

4. Locock. L, Healthcare redesign: "meaning, origins and application, Qual Saf Health Care",12, 2003, 53-57.

5. Wihlman, U., Lundborg, C. S., Holmström, I., \& Axelsson, R. (2010). Organizing vocational rehabilitation through interorganizational integration - a case study in Sweden. International Journal of Health Planning and Management, vol 26, no. 3 , pp. 169-185.

6. Cao, G., Clarke, S. \& Lehaney, B. 2001, A critique of BPR from a holistic perspective, Business Process Management Journal, vol. 7, no. 4, 332-339.

7. Rehder.S," Creating sustainable process management in healthcare", Master of Science Thesis in Quality and Operations Management, Department of Technology Management and Economics, Chalmers University of Technology,2012.

8. Kontio, J. 2007, Business process re-engineering: a case study at Turku University of Applied Sciences, Proceedings of European and Mediterranean Conference on Information Systems (EMCIS2007), 2007.

9. Yarmohammadian.M, Ebrahimipour.H, DoostyDeveloping.F, “an integrated business process management and lean model for improving quality of services in teaching hospitals", International Journal of Health System and Disaster Management , 1, 4, 2013.

10. McNulty, T., \& Ferlie, E. Process Transformation: Limitations to Radical Organizational Change 
within Public Service Organizations. Organization Studies, vol. 25, no. $8,2004$.

11. Connell .T, I Ben-Tovim.D, McCaughan. B, Szwarcbord. M , M McGrath. K,"Health services under siege: the case for clinical process redesign", MJA, Volume 188 Number $6 \cdot 17$ March ,2008.

12. Palmberg, K. Experiences of implementing process management: a multiple-case study. Business Process Management Journal, vol. 16, no. 1,2010 .

13. Minvielle.w, Waellib.M, Sicottec.C, Kimberl.j,"Managing customization in health care: A framework derivedfrom the services sector literature", Health Policy $117,2014$.

14. P. Mella The Holonic revolution: holons, holarchies and holonic networks: the ghost in the production machine Pavia University Press, Pavia, Italy 2009.

15. Seyedhoseini,S.M.,Gholamian,M.R., Movassagh,A.r."An adaptive agent based model for improving agility indicators in JIT manufacturing systems", Kuwait journal of science \& engineering, no.2b-vol.38,2011.

16. M. Calabrese, A. Amato, V. Di Lecce, V. Piuri ,'Hierarchical-granularity holonic modelling", Journal of Ambient Intelligence and Humanized Computing, Springer, 1(3), 2010.

17. B Hussein, H Bazzi, A Dayekh, W Hassan, Critical analysis of existing business process reengineering models: towards the development of a comprehensive integrated model Journal of Project, Program \& Portfolio Management 4 (1), 30-40, 2013.

18. Gotti.v, Giret.a, A multi agent methodology for Holonic Manufacturing Systems, springer, 2008.

19. Cristina Morariu, Octavian Morariu, Theodor Borangiu, Customer order management in service oriented holonic manufacturing, Computers in Industry, Volume 64, Issue 8, 2013.

20. Yong Ching Lim, Towards Holonic Academia: The theoretical framework and Literature review, Journal of Education and Practice, 2012.

21. Abdoos .M, Mozayani N, L.C. Bazzan A, "Holonic multi-agent system for traffic signals control, Engineering Applications of Artificial Intelligence, Volume 26, Issues 5-6, 2013.

22. Ounnar. F, Naamane. A, Pujo. P, M'Sirdi N, A conceptual definition of a holonic processing framework to support the design of information fusion systems, Energy Procedia, Volume 42, 2013.
23. Sun, Hongyi; Venuvinod, Patri K. Technovation : the international journal of technological innovation, entrepreneurship and technology management.- Amsterdam ,vol. 16, no. 1, pp. 93113,2001 . 